A.G. Mamalis, A. Markopoulos and D.E. Manolakos

\title{
Micro and nanoprocessing techniques and applications
}

ABSTRACT. Nanotechnology is a rapidly growing field with many and important applications. Technologies associated with this field aim towards the manufacture of improved quality products that are suitable for devices that are lighter, faster, more reliable, efficient and safer and in those respects more economical for sophisticated industrial products that perform better and are more advantageous in many other ways than any other devices manufactured so far. The realization of this production is achieved through processing techniques as sophisticated the devices themselves, either specially designed for this technological field or suitably altered processing for manufacturing large-scale super-finished products or miniaturized devices. Some of these micro and nanoprocesses, under the general term ultraprecision processes, are outlined in this paper. Furthermore, some important application fields in current and future technologies are reported in order to specifically indicate the great impact of these processes in today's industry, which also shall lead to the emerging technologies of tomorrow.

Nanotechnology Perceptions 1 (2005) 31-52

Nonsubscribers: purchase individual article 